\title{
PARAMETRIC EXCITATION OF KINETIC ION-ION HYBRID MODES IN A MULTI-ION SPECIES PLASMA
}

BY

M. ONO, R, P, H. CHANG, AND M. PORKOLAB

\section{PLASMA PHYSICS LABORATORY}

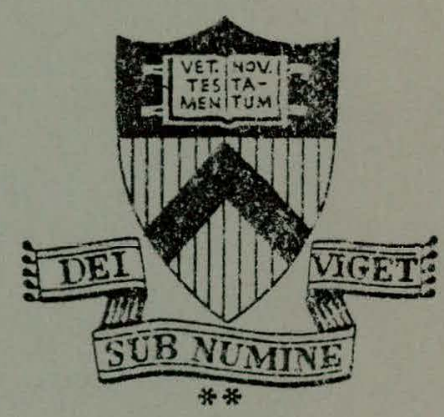

\section{PRINCETON \\ UNIVERSITY PRINCETON, NEW JERSEY}

This work was supported by U. S. Energy Research and Development Administration Contract E(1.1-1)-3073. Reproduction, translation, publication, use and disposal, in whole or in part, by or for the Uniteci States Government is permitted. 


\section{DISCLAIMER}

This report was prepared as an account of work sponsored by an agency of the United States Government. Neither the United States Government nor any agency Thereof, nor any of their employees, makes any warranty, express or implied, or assumes any legal liability or responsibility for the accuracy, completeness, or usefulness of any information, apparatus, product, or process disclosed, or represents that its use would not infringe privately owned rights. Reference herein to any specific commercial product, process, or service by trade name, trademark, manufacturer, or otherwise does not necessarily constitute or imply its endorsement, recommendation, or favoring by the United States Government or any agency thereof. The views and opinions of authors expressed herein do not necessarily state or reflect those of the United States Government or any agency thereof. 


\section{DISCLAIMER}

Portions of this document may be illegible in electronic image products. Images are produced from the best available original document. 


\section{NOTICE}

This report was prepared as an account of work sponsored by the United States Govermment. Neither the United States nor the United States Energy Research and Development Administration, nor any of their employees, nor any of their contractors, subcontractors, or their employees, makes any warranty, express

or implied, or assumes any legal liability or responsibility for the accuracy, completeness or usefulness of any information, apparatus, product or process disclosed, or represents that its use would not infringe privately owned rights.

Printed in the United States of America.

Available from

National Technical Information Service

ט. S. Department of Commerce 5285 Port Royal Road

\section{Springfiela, Virginia 22.151}

Price: Printed Copy $\$ *_{\text {; Microfiche } \$ 3.00}$

$$
\begin{gathered}
\text { *Pages } \\
1-50 \\
51-150 \\
151-325 \\
326-500 \\
501-1000
\end{gathered}
$$

NTIS

\section{Selling Price}

$\$ 4.00$

5.45

7.60

10.60

13.60 
Parametric Excitation of Kinetic Ion-Ion Hybrid Modes in a Multi-Ion Species Plasma

\author{
M. Ono, R. P. H. Changt, and M. Porkolab \\ Plasma Physics Laboratory, Princeton University \\ Princeton, New Jersey 08540
}

February 1977

PPPL-1325

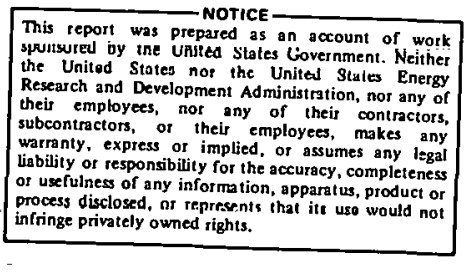


Parametric Excitation of Kinetic Ion-Ion

Hybrid Modes in a Multi-Ion Species Plasma*

M. Ono, R. P. H. Chang, ${ }^{+}$and M. Porkolab

Plasma Physics Laboratory, Princeton University,

Princeton, New Jersey 08540

\begin{abstract}
Parametric excitation of the ion-ion hybrid modes in a multi-ion species plasma is observed. The modes are excited when the pump frequency is near the sum of the ion cyclotron frequencies. The dispersion relation and the threshold for excitation are measured.
\end{abstract}


Although predicted by theory, up until now, to our knowledge, no experimentall verification of the parametric instabili-ty near the ion cyclotron frequency has been reported. ${ }^{1}$ In this letter we wish to report experimental observation of the kinetic ion-ion hybrid parametric instability in a two ion species plasma. Such an instability may occur, for example, during radio frequency heating near the ion cyclotron frequency of a deuterium-tritium fusion reactor plasma. ${ }^{1}$ since parametric instabilities may produce anomalous absorption of the incident microwave power, it is of importance to understand under what conditions they may occur.

The present instability is finduced mainly by the reliative polarization drift between the ions of different species. Although the electron drift is comparable in strength to ion drift, theory shows that for hot electrons its contribution to driving the instability is negligible. ${ }^{1}$ The resulting decay waves propagate in the direction of the pump electric field, perpendicularly to the external magnetic ifield. In a two ion species plasma, the dispersion relation of electrostatic waves predicts two propagation modes, one above each ion cyclotron frequency. The real part of this dispersion relation in a plasma: with hot electrons. and cold ions is given by the following expression:

$$
1+\frac{1}{k^{2} \lambda_{D e}{ }^{2}}-\frac{\omega_{p 1}^{2}}{\omega^{2}-\Omega_{1}{ }^{2}}-\frac{\omega_{\mathrm{p} 2}^{2}}{\omega^{2}-\Omega_{2}{ }^{2}}=0 .
$$


Here $\omega$ and $k$ are the wave frequency and wave number, $\Omega_{1}$ and $\Omega_{2}$ are the ion cyclotron frequencies of each ion species, $\omega_{\mathrm{p} 1}$ and $\omega_{\mathrm{p} 2}$ are the ion plasma frequencies, and $\lambda_{D e}$ is the electron Debye length. In contrast to the well known Buchsbaum ion-ion hybrid modes (which occurs in cold electron plasma) ${ }^{2}$, we shall call the present modes "kinetic ion-ion hybrid modes" (since these occur in a hot electron plasma). Furthermore, decay into these two modes may occur for a large range of plasma parameters. Writing the dispersion relation for each of the decay waves, $\omega_{1}(k)$ and $\omega_{2}(k)$, and using the selection rules, $\omega_{0}=\omega_{1}+\omega_{2}$, and $k_{0}=k_{1}+k_{2}=0$ (so that $\mathrm{k}_{1}=-\mathrm{k}_{2}$ ) the following relation is obtained for the lower sideband, $\omega_{2}\left(k_{2}\right)$ :

$$
\left(\omega_{2}^{2}-\Omega_{1}^{2}\right)\left[\left(\omega_{0}-\omega_{2}\right)^{2}-\Omega_{1}^{2}\right]-\frac{\mathrm{N}_{1} \mathrm{~m}_{2}}{\mathrm{~N}_{2} \mathrm{~m}_{1}}\left(\omega_{2}^{2}-\Omega_{2}^{2}\right)\left[\Omega_{2}^{2}-\left(\omega_{0}-\omega_{2}\right)^{2}\right]=0 .
$$

Here, $\mathrm{N}_{1}$ and $\mathrm{N}_{2}$ are the relative ion concentrations, and $\mathrm{m}_{1}$ and $\mathrm{m}_{2}$ are the masses of the two ion species with $\mathrm{m}_{2}$ being the mass of lighter ion species. We note that for a given pump frequency and ion species, the decay wave frequency depends only upon the relative ion concentration ratio and the magnetic field strength, and is independent of the wave number. Hence, it will be relatively easy to check experimentally the selection rules through Eq. (2). The threshold for exciting this instability in a finite length system is given by the following expression 3,4 :

$$
\gamma_{0}^{2}=v_{1} v_{2}\left[\frac{\pi^{2}}{4 L^{2}}+\frac{1}{4}\left(\frac{\Gamma_{1}}{v_{1}}+\frac{\Gamma_{2}}{v_{2}}\right)^{2}\right]
$$


where

$$
\dot{\gamma}_{0}^{2}=\frac{k^{2} u^{2}\left|x_{1}-x_{2}\right|^{2}}{4 \frac{\partial \varepsilon}{\partial \omega_{1}} \frac{\partial \varepsilon}{\partial \omega_{2}}}
$$

is the grow.th rate in a uniform plasma. ${ }^{1}$ Here $L$ is the length of the system, $V_{1,2}$ are the group velocities of the two decay waves, $\Gamma_{1,2}$ are their damping rates, $x_{1}=x(\omega)$ and $x_{2}=x\left(11-{ }^{\prime \prime}\right)$ are the ion susceptibilities of the lighter mass ion species, and: $\mathrm{U} \approx \mathrm{cE} \omega_{0}{ }^{\Omega}{ }^{\prime} \mathrm{D}\left(\omega_{0}^{2}-\Omega_{2}^{2}\right)$ is the relative polarization arift of ions (where we assumed $\Omega_{1} \ll \Omega_{2}$ ). 1

The experiments were performed in Princeton's L-4 device. A schematic of the experimental apparatus is shown in Fig. I. A hot electron plasma was produced by a tungsten filament source. 5 The range of experimental parameters were as follows: the magnetic field, $\mathrm{B}_{0} \leqslant 4.2 \mathrm{kG}$, the plasma density, $\mathrm{N}_{0}=10^{9} \approx 10^{10} \mathrm{~cm}^{-3}$ the temperatures, $\mathrm{T}_{\mathrm{e}}=3-5 \mathrm{eV}, \mathrm{T}_{i}<0.1 \mathrm{eV}$, and neutral gas filling pressure in the experimental region, $P \cong 1.0 \times 10^{-4}$ Torr. A plasma with desired ion concentration ratio was produced by properly adjusting the inflow of neutral gas 1 and 2 . The relative ion: concentration was measured by combined spectroscopic and Langmuir probe techniques. The external rf oscillator was coupled to the rf structure through an impedance matching network. The pump electric field and the resulting decay waves which propagate in the central region of the structure were investigated by radially and axially movable rf probes and also with calibrated ceramic insulated double probes. 
The rf induction coil structure was built of copper coils embedded in a ceramic insulator and was electrostatically shielded from the plasma by a Faraday shield. The structure was aligned parallel to the magnetic field lines so that the induced electric field was perpendicular to the external dc magnetic field. We have designed the shape of the coil so that the electric field induced by it was uniform in the central region of the plasma column. Because of the low plasma density, the rf electric field generated inside the plasma was essentially the vacuum field, and could be calculated directly from Maxwell's equations. The Faraday shield isolated the plasma from the electrostatic field produced by the inductive potential drop along the coil. In order to determine the exact nature of the pump electric field, we have searched for short wavelength electrostatic waves at the pump frequency, using interferometric techniques. However, our search proved negative, and we concluded that the pump electric field could be correctly described in the dipole approximation, namely $\mathrm{k}_{0}=0$.

As the pump power was increased above certain threshold level, we observed a sudden onset of parametric decay. A typical decay frequency spectrum obtained in a helium-neon plasma is shown in Fig. 2(a). (We note that similar decay waves were also observed in helium-argon or helium-krypton plasmas.) The subsequent data presented in this paper were obtained in a helium-neon plasma. As shown in Fig. 2 (a) the decay frequencies were observed to occur above each ion cyclotron frequency, and varied with the magnetic field and relative ion concentration ratio. From the variation of the decay frequencies with magnetic 
field strength, the dependence of the frequencies upon ion concentration ratio, and wavelength measurements with interferometric techniques, the decay waves. were identified to be the kinetic ion-ion hybrid waves.

The theoretical dispersion curves for the two kinetic ion-ion hybrid modes, in a $4: 6 \cdot \mathrm{He}-\mathrm{Ne}$ ion mixture, as predicted by Eq. (1), is shown by the solid curves in Fig. 2. In the same figure, the dots show the experimentally measured values of $\omega(k)$, as determined by interferometric measurements of the wave numbers of the decay waves. We believe that this is the first experimental observation of the kinetic ion-ion hybrid modes. We see a good agreement between the experimentally measured values and cold plasma theory, except near the harmonics of the ion cyclotron frequencies. In order to explain this deviation from the cold ion plasma theory, we have numerically calculated the hot plasma electrostatic wave dispersion relation, including finite ion Larmor radius effects. In Fig. 2, we show such theoretical plots (indicated as dotted lines) for different ion temperatures which may be expected in our device. We see that the best agreement between theory and experiment is obtained by assuming $\mathrm{T}_{i}: 1 / 40 \mathrm{eV}$ (or $\mathrm{T}_{i}$ approximately equal to room temperature). We note that in two ion species plasmas the gap in the dispersion curves near the second ion cyclotron harmonic frequency is considerably enhanced by.the finite ion Larmor radius effects (especially for the lighter...species). Thus, these experiments provide an interesting technique to: estimate the low ion temperatures that characterize plasma devices such as ours. 
In Fig. 3(a), we have plotted the experimentally observed decay frequencies, normalized in a unit of helium ion cyclotron frequency, for various ratios of ion concentration as a function of magnetic field. The solid lines are the theoretical curves. We see that the experimentally observed values are again in a good agreement with the theoretical curves.

In Fig. 3(b), we show the experimentally observed threshold values of pump electric field $\mathrm{E}$ as a function of the normalized wave number, $\mathrm{kCs} / \Omega_{\mathrm{He}}$. The theoretical threshold curve for the decay instability was calculated from Eq. (3) using electron Landau damping, ion-ion collisions among the different ion species and ion-neutral collisions in the imaginary parts, and finite ion Larmor radius effects in the real part of the plasma dielectric constant. For our experimental parameters, ion Landau damping and ion cyclotron damping are negligible. The ion-ion collisions among different ion species become significant for relatively high density cold ion plasma. For our experimental parameters, their contribution is comparable to that of the ion-neutral collisions, and hence, were appropriately included in the threshold calculations. The comparison of the experimentally measured values (using the calibrated rf probe) with the theoretically obtained values are in good agreement, well within the experimental uncertainty which is typically a factor of 2 . We note that the systematic deviation between theory and experiment for $\mathrm{kCs} / \Omega_{\mathrm{He}} \geq 2$ may be due to viscous ion damping, ${ }^{6}$ which was not included in our calculations. 
In conclusion, we have observed two ion species parametric instabilities near the ion cyclotron frequency. The measured dispersion relationship shows a good agreement with that of theoretical prediction of kinetic ion-ion hybrid mode. The dependence of wave matching conditions upon ion concentraion ratio and magnetic field are in good quantitative agreement. The obsenved thresholds are also found to be consistant with the theory. The saturation mechanism and the associated plasma. heating are now under detailed study.

\section{ACKNOWLEDGMENTS}

We thank P. Bellan, L. Johnson, and E. Hinnov for helpful discussions, and J. Johnson, J. Taylor, A. Sivo, and R. McWilliams for technical assistance. 


\section{REFERENCES}

*This work was supported by the U. S. Energy Research and Development Administration, Contract E(11-1)-3073.

†Permanent address: Bell Laboratories, Murray Hill, New Jersey 07974 .

${ }^{1}$ J. L. Sperling and F. W. Perkins, Phys. Fluids 17, 1857 (1974). ${ }^{2}$ S. J. Bucksbaum, Phys. Fluids 3,418 (1960).

3. Porkolab and R. P. H. Chang, Phys. Fluids 13, 2054 (1970).

4. Pesme, G. Laval, and R. Pellat, Phys. Rev. Lett. 31, 203 (1973) .

${ }^{5}$. Limpaecher and K. R. Mackenzie, Rev. Sci. Instrum. 44 , 726 (1973).

${ }^{6}$ M. Porkolab, Phys. Fluids 11,834 (1968). 


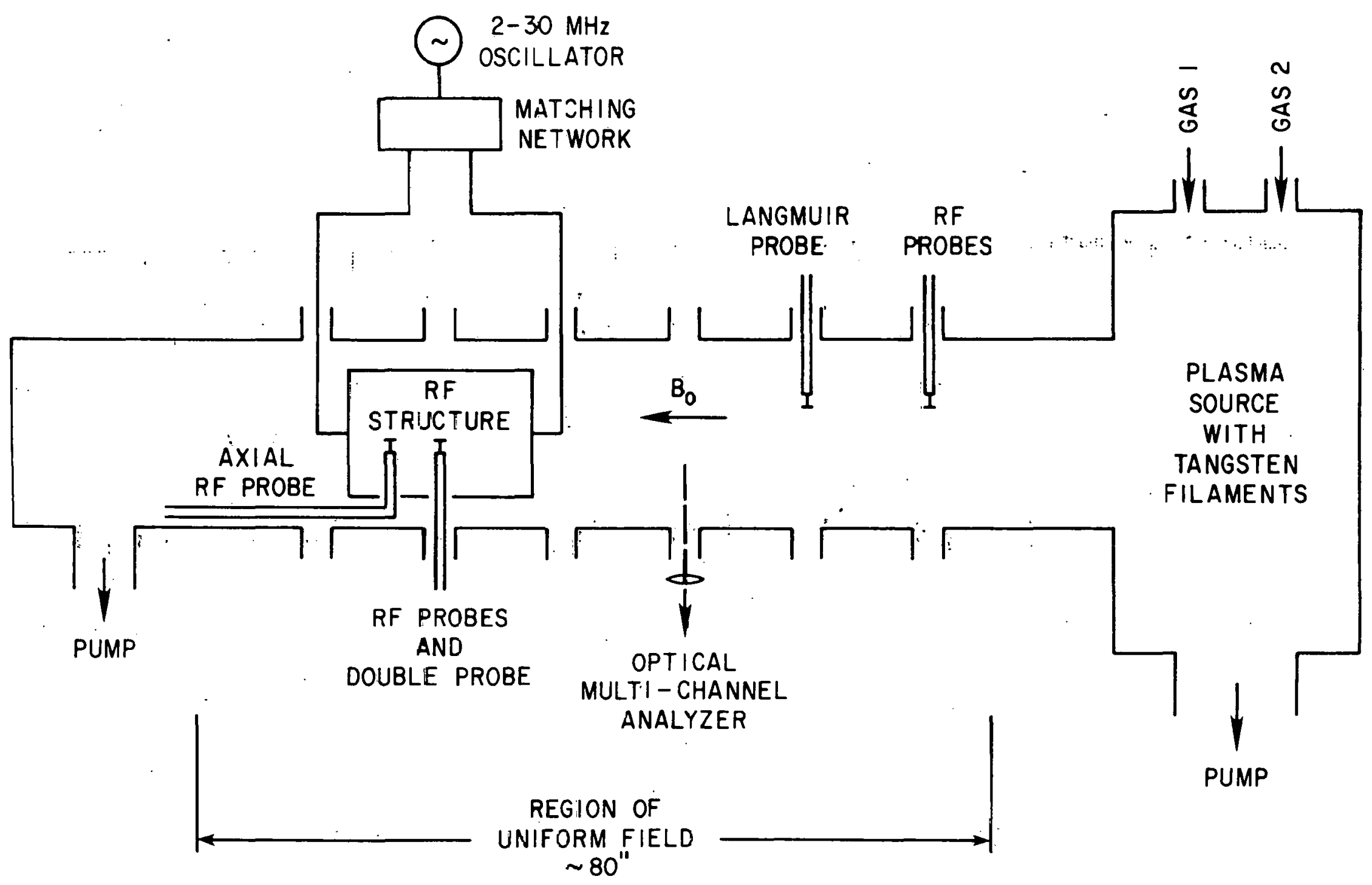

Fig. 1. Simplified schematic of the experimental set-up. 


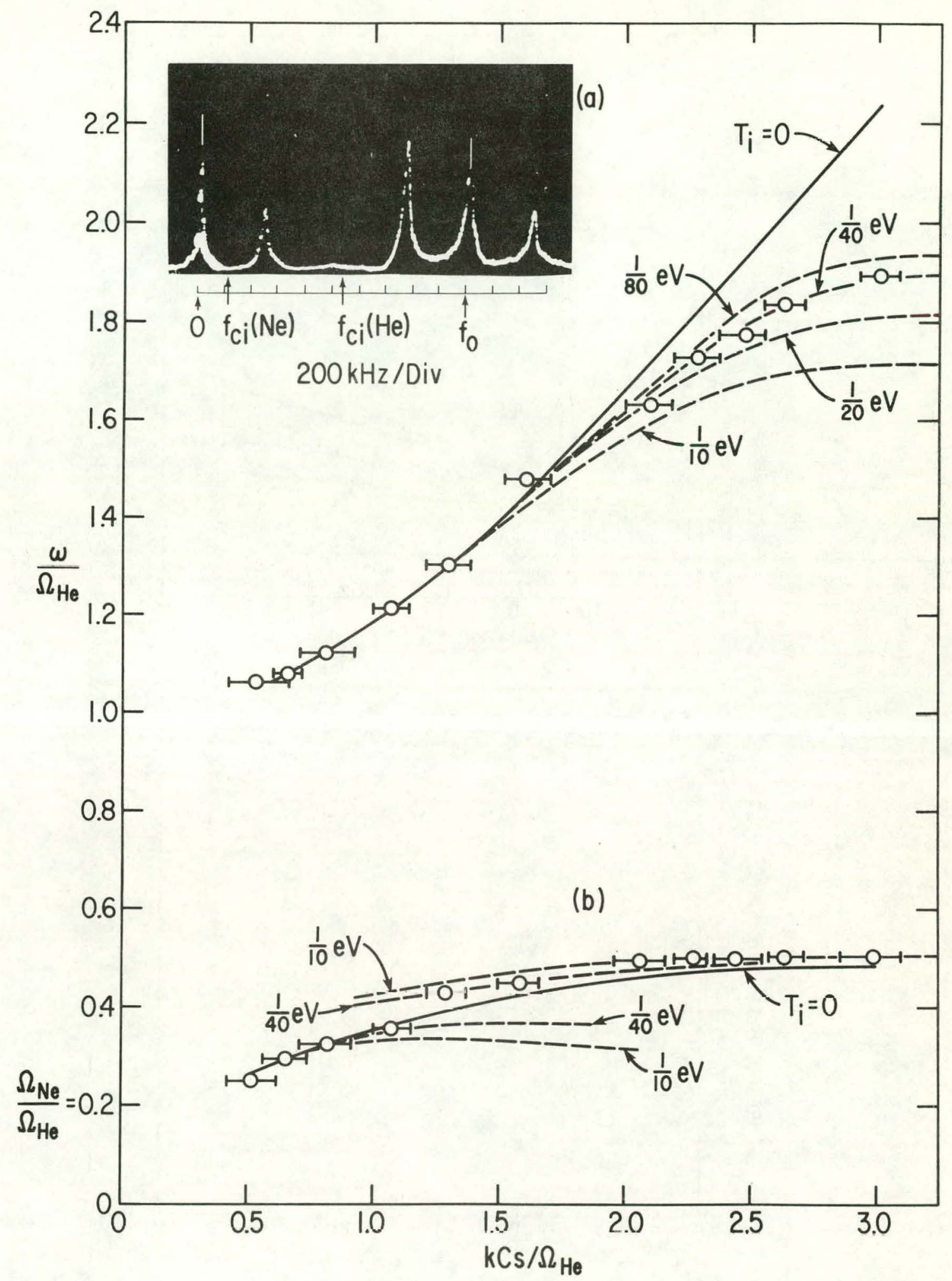

772049

Fig. 2. (a) Parametric Decay Spectrum. $f_{\mathrm{Ci}}(\mathrm{He}), \mathrm{f}_{\mathrm{Ci}}(\mathrm{Ne})$ are the helium, neon ion cyclotron frequencies. $\left(\mathrm{B}_{0}=2.9 \mathrm{kG}\right.$, $\left.\mathrm{f}_{0}=2 \mathrm{MHz}, \mathrm{He}: \mathrm{Ne}=4: 6.\right)$ (b) Dispersion curve of kinetic ionion hybrid mode. Solid and dashed curves, theory. (He:Ne $=4: 6$, Cs is the helium acoustic speed.) Circles are the experimentally measured values. 

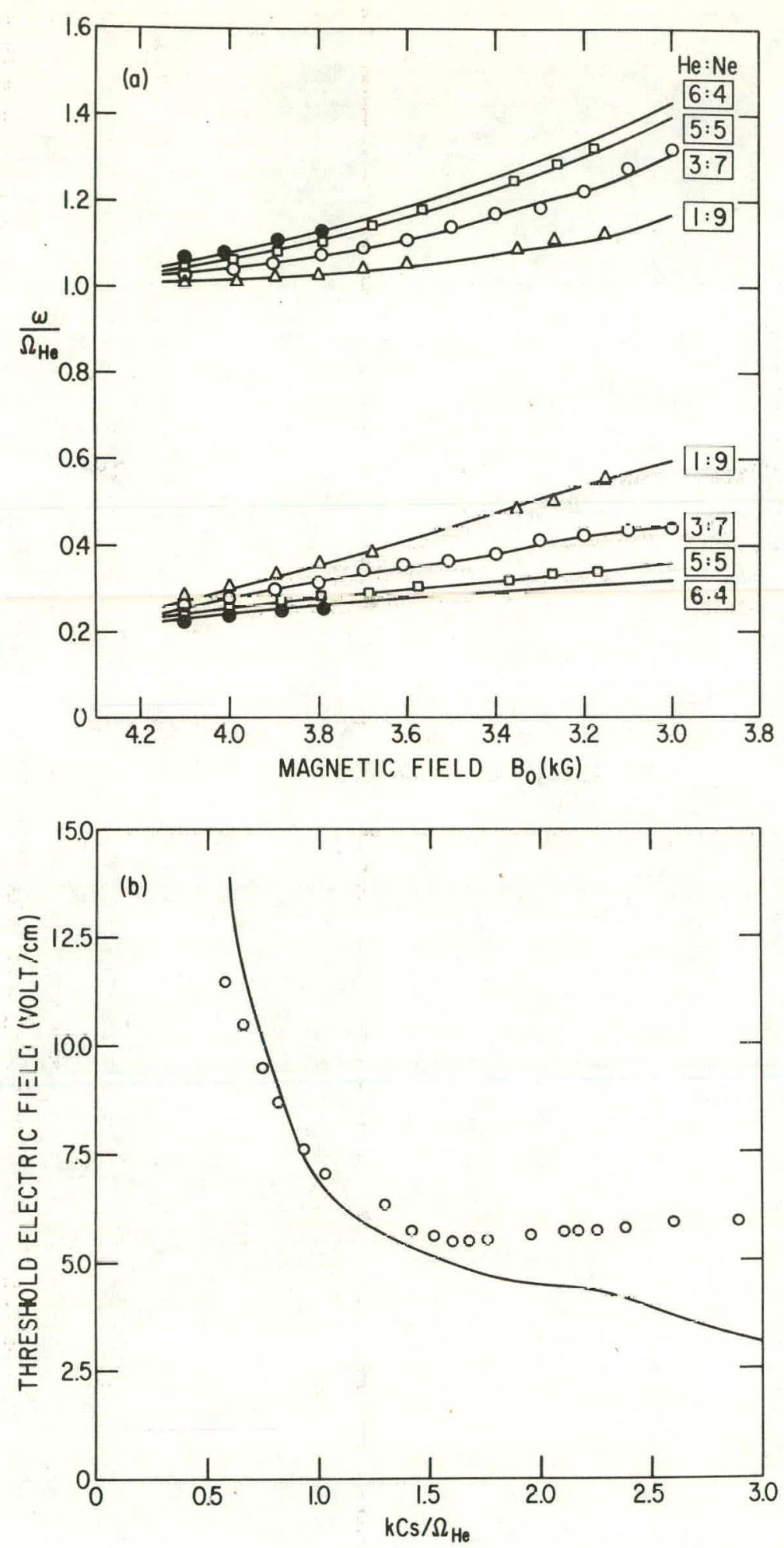

Fig. 3. (a) Decay wave frequency versus magnetic field for various ion concentration ratios. Circles and dots are the experimentally measured values, and the solid lines correspond to theory. $f_{0}=2 \mathrm{MHz}$. (b) Threshold value of pump electric field versus wave number of decay waves. Solid curve corresponds to theory ( He: $\mathrm{Ne}=4: 6)$. 